\title{
Muon-spin-relaxation studies on the heavy-fermion system with non-Fermi-liquid behavior $\mathrm{CeCu}_{5.9} \mathrm{Au}_{0.1}$
}

\author{
A. Amato, R. Feyerherm, F.N. Gygax, and A. Schenck \\ Institute for Particle Physics, ETH Zürich, CH-5232 Villigen PSI, Switzerland \\ H.v. Löhneysen and H.G. Schlager \\ Physikalisches Institut, Universität Karlsruhe, Engesserstrasse 7, D-76128 Karlsruhe, Germany
}

(Received 14 February 1995)

\begin{abstract}
Positive muon-spin-relaxation ( $\mu \mathrm{SR}$ ) measurements have been carried out down to $20 \mathrm{mK}$ on a single crystal of the heavy-fermion system $\mathrm{CeCu}_{5.9} \mathrm{Au}_{0.1}$, which exhibits non-Fermi-liquid behavior. Whereas preliminary $\mu \mathrm{SR}$ studies on the isostructural compound $\mathrm{CeCu}_{5} \mathrm{Au}$ confirms the previously reported development of an incommensurate antiferromagnetic state for this Au concentration, the zero-field data on $\mathrm{CeCu}_{5.9} \mathrm{Au}_{0.1}$ show no sign of magnetic ordering down to the lowest temperature investigated, setting an upper limit of $0.003 \mu_{B}$ for a hypothetical static Ce moment. This observation by a microscopic technique supports the assertion that non-Fermi-liquid behavior is observed in Kondo lattices when magnetic order is homogeneously suppressed to zero temperature, i.e., at a quantum phase transition between magnetic and nonmagnetic ground states.
\end{abstract}

The paramagnetic ground state of heavy-fermion (HF) compounds is often interpreted in the framework of the Landau-Fermi-liquid theory, where the properties of the strongly interacting electron system are described by those of a system of noninteracting strongly renormalized quasiparticles. ${ }^{1}$ Such a ground state, which was once thought to be predominant among the HF compounds, appears now to be, at best, an exception to the rule. This drastic modification of the phenomenological classification of HF compounds is mainly based on the detection by muon-spin-relaxation ( $\mu \mathrm{SR})$ spectroscopy of weak static magnetism in systems which were long believed to behave like Fermi-liquid paramagnets. ${ }^{2}$ This weak magnetism appears when the local $f$-spin degree of freedom has been transferred to the conduction electrons by means of the Kondo effect and is ascribed to the residual interactions between the renormalized quasiparticles (i.e., the low-energy excitations of the interacting system). ${ }^{3}$

Among the Ce-based $\mathrm{HF}$ compounds, $\mathrm{CeCu}_{6}$ plays a unique role being one of the very last Fermi-liquid paramagnets. ${ }^{4}$ But despite its paramagnetic ground state $\mathrm{CeCu}_{6}$ appears to be close to a magnetic anomaly, with short-range dynamical magnetic correlations developing below $10 \mathrm{~K} .{ }^{5}$ Moreover it has been shown that the substitution of $\mathrm{Au}$ or $\mathrm{Ag}$ for $\mathrm{Cu}$ leads to the occurrence of magnetic ordering, ${ }^{6}$ which is interpreted in the frame of the Doniach picture for Kondo lattices with strong exchange interaction between the localized $f$ electrons and the conduction electrons. ${ }^{7}$ The substitution of $\mathrm{Cu}$ by atoms of larger atomic radius ( $\mathrm{Au}$ or $\mathrm{Ag}$ ) expands the lattice and therefore weakens the exchange interaction, leading to the stabilization of the local $f$ magnetic moment against the formation of local Kondo singlets. Above a critical $\mathrm{Au}$ or $\mathrm{Ag}$ concentration $x_{c}$, antiferromagnetism occurs when the condensation energy due to the coupling of neighboring $f$ moments via Ruderman-Kittel-KasuyaYosida (RKKY) interactions is larger than the energy gained by forming local Kondo singlets. The decrease of the Néel temperature with hydrostatic pressure confirms this picture. ${ }^{8}$ For alloys with the critical concentration $x_{c}$ one therefore expects that both RKKY and Kondo interactions have the same magnitude. Recently the specific heat $C$ and the resistivity $\rho$ of $\mathrm{CeCu}_{6-x} \mathrm{Au}_{x}$ with $x=x_{c}=0.1$ have been shown to exhibit non-Fermiliquid behavior $\left[C / T \propto-\ln \left(T / T_{o}\right), \Delta \rho \propto T\right.$, where $\Delta \rho$ is the temperature-dependent part of $\rho$ (Ref. 9)]. This was attributed to the complete suppression to zerotemperature of the magnetic order transition due to the competition with the Kondo effect. Nevertheless the apparent absence of magnetic order for finite temperatures was only deduced from macroscopic studies, which in the field of heavy-fermion physics (keeping in mind the weak magnetism phenomena detected by microscopic techniques) is not sufficient. We have therefore undertaken $\mu \mathrm{SR}$ studies on some $\mathrm{CeCu}_{6-x} \mathrm{Au}_{x}$ single-crystal samples in order to test the complete absence of static magnetism for $x \rightarrow x_{c}$.

Single crystals with $x=0.1$ and $x=1$ were grown with the Czochralski method as described previously. ${ }^{10}$ The $\mu$ SR experiments were carried out at the Paul Scherrer Institute (Villigen, Switzerland), using a ${ }^{3} \mathrm{He}-{ }^{4} \mathrm{He}$ dilution refrigerator and a He-flow cryostat, on the $\mu \mathrm{SR}$ dedicated beam line ( $\pi \mathrm{M} 3$ ) of the PSI-600 $\mathrm{MeV}$ proton accelerator. In order to avoid a relaxing background $\mu \mathrm{SR}$ signal, the samples were glued onto high-purity silver holders. Zero-field (ZF) measurements have been performed with the initial muon polarization $\left[\mathbf{P}_{\mu}(0)\right]$ along the $b$ axis (orthorhombic notation). Such an experimental configuration was specifically chosen by considering the incommensurate magnetic structure determined by neutron diffraction for a sample with $x=0.5$ [wave vector $\mathbf{q}=( \pm 0.59,0,0)$ and moments aligned along the $c$ axis $^{11}$ ] and that the $\mu^{+}$is located at the $b$ site (Wyckoff notation) as unambiguously determined in $\mathrm{CeCu}_{6} \cdot{ }^{12} \mathrm{Cal}-$ culations show that in this case the total dipolar field at 
the $\mu^{+}$site produced by the magnetically ordered neighboring $\mathrm{Ce}$ atoms forms an angle close to $90^{\circ}$ with the $b$ axis and will cause, in the case $\mathbf{P}_{\mu}(0) \| b$ axis, an easily detectable oscillating $\mu \mathrm{SR}$ signal. ${ }^{13}$

For $\mathrm{CeCu}_{5} \mathrm{Au}(x=1)$, the $\mathrm{ZF} \mu \mathrm{SR}$ data clearly reveal the antiferromagnetic transition by the occurrence of spontaneous Larmor frequencies below $T_{N} \simeq 2.3 \mathrm{~K} .{ }^{13}$ In the magnetic phase, the time evolution of the muon polarization can be well described by a damped $J_{0}$ Bessel function (see Fig. 1) which is theoretically expected for an incommensurate magnetic structure described by one q-wave vector. ${ }^{14,13}$ Assuming the above described incommensurate magnetic structure observed by neutron studies, a value of $\mu_{s} \simeq 0.8 \mu_{B} / \mathrm{Ce}$ for the static magnetic moment can be extracted from the $\mu$ SR data. Such a value is in fairly good agreement with the rough estimations of $0.5-1.0 \mu_{B}$ obtained by neutron studies in $\mathrm{CeCu}_{5.5} \mathrm{Au}_{0.5}{ }^{11,15}$

$\mathrm{By}$ decreasing the $\mathrm{Au}$ concentration to $x=0.1$ (i.e., the critical concentration $x_{c}$ ), the ZF $\mu$ SR measurements performed between 0.02 and $20 \mathrm{~K}$ fail to detect any clear magnetic transition down to the lowest investigated temperature. For all temperatures, the time evolution of the muon polarization (an example is shown in Fig. 2) is best described by the well known Kubo-Toyabe (KT) function: ${ }^{16}$

$$
G_{\mathrm{KT}}^{\mathrm{ZF}}(t)=\frac{1}{3}+\frac{2}{3}\left(1-\Delta^{2} t^{2}\right) \exp \left(-\frac{1}{2} \Delta^{2} t^{2}\right),
$$

where $\Delta^{2} / \gamma_{\mu}^{2}$ represents the second moment of the local field distribution at the muon site $\left(\gamma_{\mu}\right.$ is the gyromagnetic ratio of the muon). Such a depolarization function (which possesses an initial Gaussian character for $\left.t<\Delta^{-1}\right)$ is characteristic of a paramagnetic state where the muon depolarization is solely due to the dipolar fields from the nuclear moments (mainly ${ }^{63} \mathrm{Cu}$ and ${ }^{65} \mathrm{Cu}$ ) which appear randomly oriented and static within the $\mu^{+}$lifetime. Although the $\mathrm{Au}$ ions substituting the $\mathrm{Cu}$ ions expand the lattice and possess a strongly reduced nuclear moment, the measured values of the depolarization rate

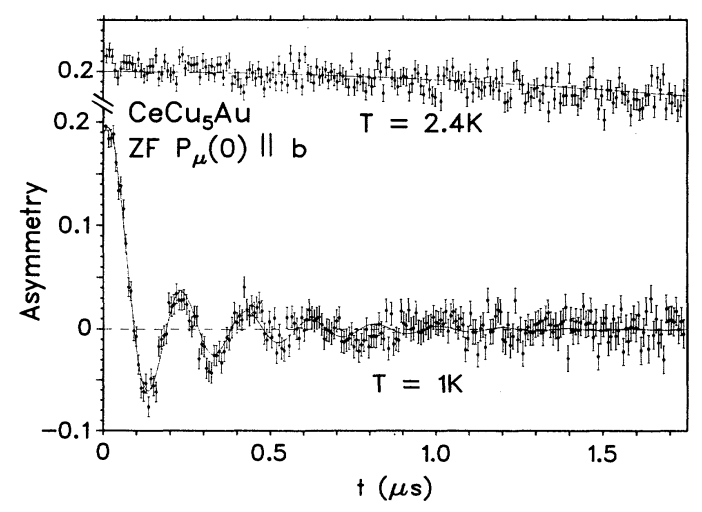

FIG. 1. Zero-field $\mu$ SR spectra obtained above and below the Néel temperature in $\mathrm{CeCu}_{5} \mathrm{Au}$. The initial muon polarization was along the orthorhombic $b$ axis. For $T=1 \mathrm{~K}$, the line represents a fit of an exponentially damped $J_{0}$ Bessel function to the data.

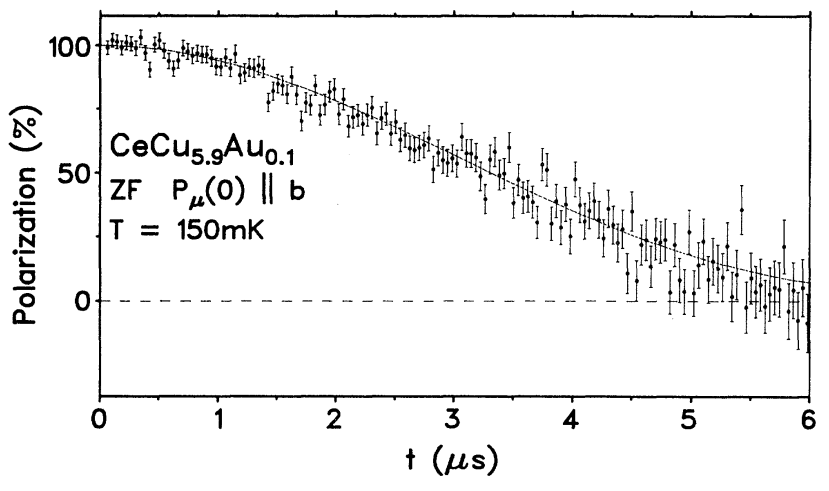

FIG. 2. Example of zero-field $\mu$ SR spectra obtained in $\mathrm{CeCu}_{5.9} \mathrm{Au}_{0.1}$ for $T=0.15 \mathrm{~K}$ with the initial muon polarization along the $b$ axis. The line represents a fitted KT function.

$\Delta$ of the $\mathrm{KT}$ function are identical in $\mathrm{CeCu}_{5.9} \mathrm{Au}_{0.1}$ and in $\mathrm{CeCu}_{6}$ (see Fig. 3). This similarity results from the fact that for $x \leq 1$ the Au atoms exclusively occupy the $\mathrm{Cu}(2)$ sites, ${ }^{18}$ which are not located in the vicinity of the $\mu^{+}$site $^{19}$ and therefore only marginally contribute to the field distribution at the $\mu^{+}$site. The main components of this field distribution arise firstly from the $\mathrm{Cu}$ atoms located at the $\mathrm{Cu}(5)$ sites and secondly from those located at the $\mathrm{Cu}(1)$ sites. Assuming the small local lattice relaxation around the $\mu^{+}$deduced from the Knight-shift data, ${ }^{12}$ one calculates for pure $\mathrm{CeCu}_{6}$ a theoretical value of $\Delta=0.246 \mu \mathrm{s}^{-1}$ for the $\mu^{+}$-depolarization rate at the $b$ site, which is in fairly good agreement with the measured value. With the observed linear variation of the lattice parameters between $x=0$ and $x=1,{ }^{11}$ we calculate for $x=0.1$ an almost negligible decrease of $0.4 \%$ of the second moment of the local field distribution at the $\mu^{+}$site, which corresponds to a change of the muon depolarization rate smaller than our experimental resolution.

Regardless of the subtle contributions to the depolarization rate, the complete absence of spontaneous Larmor

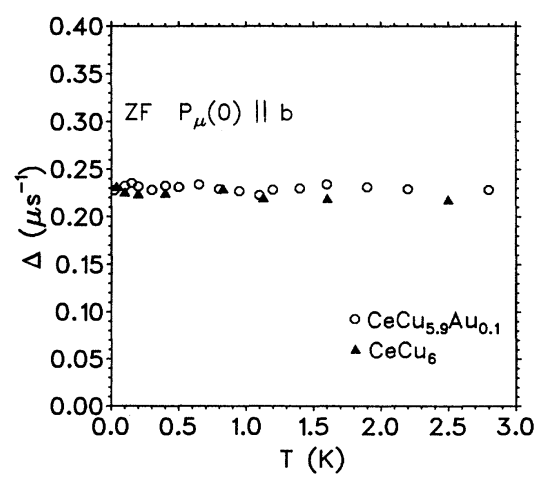

FIG. 3. Temperature dependence of the zero-field depolarization rate in $\mathrm{CeCu}_{5.9} \mathrm{Au}_{0.1}$ and pure $\mathrm{CeCu}_{6}$. The error bars are smaller than the data-point size. Data for $\mathrm{CeCu}_{6}$ after Ref. 17. 
frequencies in the first place and/or of a clear change of behavior of the depolarization rate in the $\mu \mathrm{SR}$ signal of $\mathrm{CeCu}_{5.9} \mathrm{Au}_{0.1}$ strongly supports a nonmagnetic ground state for this alloy. The observation of a constant depolarization rate for the whole temperature range sets an upper limit of $\Delta_{4 f} \simeq 0.10 \mu \mathrm{s}^{-1}$ for an additional depolarization which would be due to a hypothetical magnetic order of the Ce $4 f$ moments. Such a value is obtained by considering that an increase larger than $0.02 \mu \mathrm{s}^{-1}$ of the overall depolarization rate $\Delta$ would have been easily detected in the present data. Considering for the hypothetical magnetic order the incommensurate magnetic structure observed for $x=0.5$ (which, as reported above, leads to a $J_{0}$-Bessel depolarization function whose early time behavior can also be approximated by a Gaussian function), the upper limit of $\Delta_{4 f}$ represents an upper limit of $3 \times 10^{-3} \mu_{B} /$ Ce for a static $4 f$ moment. Assuming on the other hand a dense spin-glass picture, where the static $4 f$ moments are randomly oriented, an upper limit of $1.5 \times 10^{-3} \mu_{B} / \mathrm{Ce}$ is obtained. Finally, a dilute spin-glass picture, with a small concentration of large static magnetic moments, can be discarded since it would lead to a Lorentzian field distribution, ${ }^{20}$ or exponential depolarization function, which is is not observed in the present data where the early time behavior of the muon depolarization appears purely Gaussian. Therefore our measurements indicate the virtually complete absence of any magnetic transition in $\mathrm{CeCu}_{6-x} \mathrm{Au}_{x}$ for $x \rightarrow x_{c}$ This appears compatible with the suggestion that the nonFermi-liquid behavior reported recently in $\mathrm{CeCu}_{5.9} \mathrm{Au}_{0.1}$ (Ref. 9) occurs when the paramagnetic and magnetically ordered ground states are practically degenerate. In particular this unusual and intriguing behavior cannot be attributed to some sort of magnetic inhomogeneity, but points to a quantum phase transition between magnetic and nonmagnetic ground states at zero temperature.

To conclude, our $\mu \mathrm{SR}$ results confirm the occurrence of magnetic ordering (most probably with incommensurate structure) in $\mathrm{CeCu}_{5} \mathrm{Au}$. On the other hand, our studies on $\mathrm{CeCu}_{5.9} \mathrm{Au}_{0.1}$ did not reveal any indication of static magnetic order confirming the close relation between non-Fermi-liquid behavior and suppression of magnetic order.

We would like to aknowledge O. Stockert for his help with the sample preparation as well as C. Baines for his technical support during the experiment. Part of this work was supported by the Deutsche Forschungsgemeinschaft.
${ }^{1}$ N. Grewe and F. Steglich, in Handbook on the Physics and Chemistry of Rare Earths, edited by K.A. Gschneider and L. Eyring (North-Holland, Amsterdam, 1991), Vol. 14, p. 343.

2 A. Schenck, in Frontiers in Solid State Sciences, edited by L.C. Gupta and M.S. Murani (World Scientific, Singapore, 1993), Vol. Magnetism, p. 269.

${ }^{3}$ N. Grewe and B. Welslau, Solid State Commun. 65, 437 (1988).

${ }^{4}$ See, for example, A. Amato et al., J. Low Temp. Phys. 68, 371 (1987).

${ }^{5}$ L.P. Regnault et al., Phys. Rev. B 38, 4481 (1988).

${ }^{6}$ A. Germann et al., J. Phys. (Paris) Colloq. 49, C8-755 (1988); A.K. Gangopadhyay et al., Phys. Rev. B 38, 2603 (1988).

${ }^{7}$ S. Doniach, Physica B 91, 231 (1977).

${ }^{8}$ A. Germann and H.v. Löhneysen, Europhys. Lett. 9, 367
(1989).

${ }^{9}$ H.v. Löhneysen et al., Phys. Rev. Lett. 72, 3262 (1994).

${ }^{10}$ H.G. Schlager et al., J. Low Temp. Phys. 90, 181 (1993).

${ }_{11}$ A. Schröder et al., Physica B 199-200, 47 (1994).

${ }^{12}$ A. Amato et al., PSI Newsletters, Annex I, 89 (1993).

${ }^{13}$ A complete report will be published elsewhere.

${ }^{14}$ J. Major et al., Hyperfine Interact. 31, 259 (1986).

${ }^{15}$ T. Chattopadhyay et al., Z. Phys. B 80, 159 (1990).

${ }^{16} \mathrm{R}$. Kubo and T. Toyabe, Magnetic Resonance and Relaxation, edited by R. Blinc (North-Holland, Amsterdam, 1967).

${ }^{17}$ A. Amato et al., Physica B 186-188, 273 (1993).

${ }^{18}$ M. Ruck et al., Acta Crystallogr. B 49, 936 (1993).

${ }^{19}$ D.T. Cromer et al., Acta Crystallogr. 13, 913 (1960).

${ }^{20}$ R.E. Walstedt and L.R. Walker, Phys. Rev. 89, 4857 (1974). 